\title{
Theoretische und methodische Antworten der Kommunikationswissenschaft auf Herausforderungen der Digitalisierung
}

\section{Einleitung der Gastherausgeberinnen und -herausgeber}

\author{
Christian Strippel • Annekatrin Bock • Christian Katzenbach • \\ Merja Mahrt • Lisa Merten · Christian Nuernbergk • Christian Pentzold • \\ Annie Waldherr
}

(C) Springer Fachmedien Wiesbaden GmbH, ein Teil von Springer Nature 2018

Am 17. Mai 1996 wurde in Leipzig die DGPuK-Arbeitsgruppe „Computervermittelte öffentliche Kommunikation" gegründet. In einem Beitrag für den Aviso zitierte Gründungsmitglied Wolfgang Schweiger (2004) später aus dem Protokoll der konstituierenden Sitzung: „Die Arbeitsgruppe arbeitet medienspezifisch und damit teildisziplinübergreifend“, weshalb sie, so Schweiger weiter, im Grunde „die Kommunikationswissenschaft im Kleinen“ abbilde (S. 14). Der Gründung vorausgegangen war 1995 eine Diskussion in der 13. Ausgabe des Aviso über „Digitale Revolution und Kommunikationswissenschaft“. Unter dem Titel „Auf dem Wege nach Babel?" dachten Fachvertreterinnen und -vertreter darüber nach, welche Konsequenzen der „Communication Highway“, „Multimedia“ und der „Umbruch in der Medienwelt" für Kommunikationsforschung, Berufsausbildung und Berufsbilder im Medienbereich sowie für die Kommunikationspolitik haben dürfte. Für das

C. Strippel $(\bowtie)$

Institut für Publizistik- und Kommunikationswissenschaft, Freie Universität Berlin,

Garystraße 55, 14195 Berlin, Deutschland

E-Mail: christian.strippel@fu-berlin.de

A. Bock

Georg-Eckert-Institut, Leibniz Institut für Internationale Schulbuchforschung Braunschweig, Celler Straße 3, 38114 Braunschweig, Deutschland

E-Mail: bock@leibniz-gei.de

C. Katzenbach

Alexander von Humboldt Institut für Internet und Gesellschaft, Französische Straße 9, 10117 Berlin, Deutschland

E-Mail: christian.katzenbach@hiig.de

M. Mahrt

Institut für Sozialwissenschaften, Heinrich-Heine-Universität Düsseldorf,

Universitätsstraße 1, 40225 Düsseldorf, Deutschland

E-Mail: mahrt@hhu.de 
Fach selbst wurde dabei unter anderem die „Vernetzung des europäischen Wissens“ (Manfred Rühl), eine stärkere Arbeit an Begriffen wie „Medium“, ,Wirkung“, „,Realität“ und „Funktion“ (Georg Ruhrmann), ein „Brückenschlag zwischen Disziplinen, wissenschaftlichen ,communities', Konzepten/Methoden und Forschungsstrategien" (Barbara Mettler-v. Meibom) sowie ,die Entwicklung eines neuartigen Begriffs der ,Kommunikationskultur“" (Peter Glotz) gefordert.

Ziemlich genau 20 Jahre später hat Andreas Hepp (2016) mit seinem Beitrag „Kommunikations- und Medienwissenschaft in datengetriebenen Zeiten“ den Aufschlag für eine Neuauflage dieser Diskussion geliefert. Die Schlagworte mögen andere sein - nun ist von „Digitalisierung“, ,Datafizierung“ und ,,permanenter Vernetzung“ die Rede -, der Schwerpunkt der Diskussion aber ist doch ähnlich geblieben: In den Diskussionsbeiträgen von Hans-Bernd Brosius (2016), Otfried Jarren (2016) und Anna Maria Theis-Berglmair (2016) ging es im direkten Anschluss an Hepp um die Fragen, was heute noch der Gegenstand der Kommunikationswissenschaft sein kann und welche Theorien und Methoden geeignet sind, um ihn zu erforschen. In der Zwischenzeit hatte die Arbeitsgruppe „Computervermittelte öffentliche Kommunikation“ zuerst das Wort „öffentliche“ aus ihrem Namen gestrichen und dann „Computervermittelte“ durch „Digitale“ ersetzt. Wer die beiden Diskussionen von damals und heute miteinander vergleicht, wird sehen, dass sich in diesen beiden Namensänderungen die heute diskutierten Fragen für das ganze Fach widerspiegeln. Man könnte fast sagen: Die Fachgruppe ist längst nicht mehr nur das kleine Abbild der Kommunikationswissenschaft, sondern vielleicht sogar ihr Prototyp.

Vor diesem Hintergrund haben wir uns zu Beginn dieses Jahres mit einer Kollektivreplik an der aktuellen Debatte beteiligt (vgl. Strippel et al. 2018b). Hier vertraten wir die These, dass die in den anderen Beiträgen geforderte Zukunft des Faches bereits in vielfältiger Weise gegenwärtig sei, bislang jedoch vor allem an den (interdisziplinären) Rändern des Faches stattfinde. Als Belege führten wir zahlreiche Studien und Theoriearbeiten an, die aus unserer Sicht in eben jene Zukunft - oder Zukünfte - weisen, über die im Fach gerade diskutiert wird. Das Problem sei stattdessen, so unser Standpunkt, dass diese Arbeiten bislang noch nicht ausreichend wahrgenommen und gewürdigt würden. Die Herausgeberinnen und Herausgeber der Publizistik

\footnotetext{
L. Merten

Hans-Bredow-Institut, Rothenbaumchaussee 36, 20148 Hamburg, Deutschland

E-Mail: 1.merten@hans-bredow-institut.de

C. Nuernbergk

Institut für Kommunikationswissenschaft und Medienforschung, Ludwig-Maximilians-Universität München, Oettingenstraße 67, 80538 München, Deutschland

E-Mail: christian.nuernbergk@ifkw.lmu.de

C. Pentzold

Zentrum für Medien-, Kommunikations- und Informationsforschung ZeMKI, Universität Bremen, Linzer Straße 4, 28359 Bremen, Deutschland

E-Mail: christian.pentzold@uni-bremen.de
}

\author{
A. Waldherr \\ Institut für Kommunikationswissenschaft, Westfälische Wilhelms-Universität Münster, Bispinghof \\ 9-14, 48143 Münster, Deutschland \\ E-Mail: annie.waldherr@uni-muenster.de
}


nahmen diese Problembeschreibung erfreulicherweise zum Anlass, sich mit der Idee eines Themenheftes zur Debatte an uns zu wenden, um nun solchen zukunftsweisenden Ansätzen jenes Forum im (publizistischen) Kern des Faches einzuräumen, an dem es bislang noch mangelt.

Diese Einladung und die damit verbundene Aufgabe der Gastherausgeberschaft haben wir sehr gerne angenommen. Das vorliegende Themenheft ist das Ergebnis dieses Anliegens, in der Debatte um die zukünftige Orientierung und um die $\mathrm{Zu}$ kunft des Faches selbst die Ebene konkreter empirisch fundierter bzw. empirisch orientierter Arbeiten in den Mittelpunkt zu rücken. Entsprechend steht das Heft mitten in der Diskussion, was sich auch in den versammelten Beiträgen spiegelt, die nicht von fertigen Projekten, sondern aus laufenden Vorhaben berichten und weitere Perspektiven entwickeln.

Thema des Heftes bleiben die inzwischen ausführlich beschriebenen (vermeintlichen) Umwälzungen in den Gegenständen der Kommunikationswissenschaft, in den methodischen Entwicklungen, die hier und in anderen Fächern gemacht werden, und dem sich wandelnden disziplinären Feld an der Schnittstelle von Sozial-, Kultur- und Geisteswissenschaften sowie der Informatik. Die Hinwendung zu den sich damit stellenden Herausforderungen folgt grundlegenden Fragen des Faches, sie eröffnet aber auch neue Problemfelder, etwa die Automatisierung von Verfahren und die Nutzung digitaler Methoden.

Ziel dieser Ausgabe ist dabei weder die Fortführung noch der Abschluss der laufenden Diskussion, sondern ihre Ergänzung um Beispiele aus der Forschungspraxis. In dem entsprechenden Call for Papers wurden deshalb auch ,konkrete theoretisch und/oder methodisch innovative Antworten auf die in der Debatte thematisierten Herausforderungen der Digitalisierung" (Strippel et al. 2018a, S. 7) erbeten. Verbunden war damit das Anliegen, den Schwerpunkt der auch nach unserer Replik bereits fortgeführten Diskussion (vgl. Krüger und Meyen 2018) beispielhaft auf konkrete Arbeitsfelder und Studien zu legen.

So gehen Barbara Pfetsch, Maria Löblich und Christiane Eilders in diesem Heft der Frage nach, wie die theoretische Sicht auf Öffentlichkeit weiterentwickelt werden kann, um auch digitalisierte Öffentlichkeiten angemessen zu fassen. Unter dem Titel „Dissonante Öffentlichkeiten als Perspektive kommunikationswissenschaftlicher Theoriebildung " diskutieren sie Dissonanz als tragfähigen Interpretationsrahmen der Öffentlichkeitstheorie, der die derzeitigen Verhältnisse in digitalen und hybriden Medienumgebungen angemessen zu reflektieren in der Lage sei. Viel- und Unstimmigkeiten würden dabei durch einen geringen Grad an Moderation gefördert, was insbesondere in digitalen Öffentlichkeiten schwach ausgeprägt sei. Dies kann sich sowohl in einem bezugslosen Nebeneinander äußern, als auch in expliziter Gegenrede. Die Autorinnen plädieren dafür, kritische, antagonistische und partizipatorische Ansätze aus der Öffentlichkeitssoziologie und der Gegenöffentlichkeit zusammen mit Netzwerktheorien zu denken. Denn die bisherigen Theorieangebote berücksichtigten die Entstehungsbedingungen, Folgen und gesellschaftlichen Wirkungen dissonanter Öffentlichkeiten bisher nicht in ausreichendem Maße. Speziell mit Blick auf Dissonanz könnten somit verschiedene aktuelle Phänomene systematisch untersucht werden, so etwa Veränderungen der Rollen und Formen des Journalismus infolge des neuen Zuschnitts von Öffentlichkeiten, die öffentliche Resonanz auf emotionale 
und affektive Kommunikationsmodi (vgl. Papacharissi 2015) sowie technische und strukturelle Eingriffe in die Informationsverarbeitung. Pfetsch, Löblich und Eilders zeigen mit ihrem Beitrag eine nach Akteuren, Modi und Strukturen differenzierte Forschungsagenda auf. Sie gehen damit auf viele Aspekte in der bisherigen Diskussion um Zukunft und Gegenstand des Fachs ein und ergänzen einen theoretischen Bezugspunkt. Eine solche Öffentlichkeitstheorie, die stärker als bisher veränderte mediale Randbedingungen und relationale Dynamiken integriert (vgl. Klinger 2018; Neuberger 2014, 2017; Waldherr 2017), halten wir für besonders gewinnbringend.

Gegen den Ruf nach Neufassungen von Öffentlichkeitstheorien und zentralen Begriffen des Fachs wenden sich hingegen Jakob Jünger und Henriette Schade in ihrem Beitrag „Liegt die Zukunft der Kommunikationswissenschaft in der Vergangenheit? Ein Plädoyer für Konstanz statt Veränderung am Beispiel der Begriffe Öffentlichkeit und Beobachtung “. Sie nehmen die Diskussion um die Zukunft der Kommunikations- und Medienwissenschaft aus neoinstitutionalistischer Perspektive in den Blick und sehen den vielfach betonten Wandel durch Digitalisierung als Rationalitätsmythos (vgl. Meyer und Rowan 1977), mit dem das Fach auch und vielleicht vor allem auf Veränderungen der Wahrnehmung von Kommunikation und Gesellschaft reagiert, aber nicht unbedingt auf Veränderungen der Untersuchungsgegenstände an sich. Die beiden schlagen vor, in der Diskussion um das Selbstverständnis unserer Disziplin Kontinuität statt Wandel stärker in den Blick zu nehmen. Unklarheiten etwa in der Abgrenzung methodischer Verfahren wie der Inhaltsanalyse und der Beobachtung habe es auch vor der Digitalisierung schon gegeben. Dies sei also keine Besonderheit digitalisierter Forschung. Ebenso gebe es schon lange vielfältige und teilweise unklare Formen von Öffentlichkeit, auf die die Forschung aber erst jüngst im Zuge der Digitalisierung aufmerksam geworden sei (vgl. etwa Jünger 2018). Für ein Themenheft zu den Herausforderungen der Digitalisierung für die Kommunikationswissenschaft mag dies zunächst eine überraschende Perspektive sein. Gleichwohl zeigt dieser Beitrag, dass eine ungewohnte Sichtweise auf einen viel diskutierten Gegenstand zu dessen besserem Verständnis beitragen kann. Tatsächlich müssen nicht alle Begriffe neu definiert und alle Theorien neu geschrieben werden. Im Gegenteil, durch den Beitrag von Jünger und Schade bekommen wir aufgezeigt, wie hilfreich ein neuer Blick für die Lösung älterer Probleme sein kann. Dies betrifft sowohl das Verständnis zentraler Begrifflichkeiten als auch die Frage nach dem Gegenstand unseres Fachs.

Mit dem Beitrag „Journalistik praxistheoretisch betreiben: Impulse für ein $d y$ namisches Verständnis des Journalismus im Kontext seiner Neuordnung “ reagiert Christopher Buschow auf die in unserem Call for Papers gestellte Frage, welche (neuen) Konzepte sich eignen, um gegenwärtige Kommunikationspraxis unter Bedingungen der Digitalisierung angemessen zu erfassen. Wie der Titel schon verrät, schlägt er darin vor, die Journalistik um einen praxeologischen Blickwinkel zu ergänzen. Dabei tritt er nicht nur an, eine neue theoretische Perspektive neben anderen zu entwickeln, sondern sein Plädoyer geht einher mit dem Anspruch, damit auch aktuelle Entwicklungen des Journalismus besser als andere Ansätze erfassen zu können. Anhand eines vierfachen ,krisenhaften Wandels“ des Journalismus zeigt Buschow auf, wie die vier Transformationsbereiche - Abgrenzung zu anderen medialen Angeboten, technologische Neuerungen, neue Formen der Wertschöpfung, 
Legitimierung des Journalismus - ausgehend von journalistischen Praktiken verstanden werden können. Mit seinem Rückgriff auf das Feld der Praxistheorie, das insbesondere in der Soziologie und Sozialphilosophie entwickelt wurde, wendet er den in der Kommunikationswissenschaft bewährten Modus des Theorieimports an, bei dem konzeptuelle Aspekte aus anderen Zusammenhängen übernommen und für das Verständnis von Kommunikationsdynamiken und Mediensystemen adaptiert werden (vgl. Hagen et al. 2015). Der von Buschow unternommene Überblick ist aus unserer Sicht ein hilfreicher erster Entwurf, der das Potenzial hat, in seiner ,empirisch-pragmatischen“ Variante für andere Bereiche der Kommunikationsforschung und Medienanalyse mit Gewinn ausbuchstabiert zu werden. Dabei liegt der eigentliche Ertrag weniger in der theoretischen Konzeptualisierung sich stark verändernder kommunikationswissenschaftlicher Gegenstände, sondern vielmehr im empirischen Anspruch der Praxistheorien, beobachtbare und beschreibbare Sozialphänome ausgehend von der zeitlich, räumlich und material gebundenen Vollzugswirklichkeit zu rekonstruieren (vgl. Bourdieu 1998; Gherardi 2012; Schäfer et al. 2015). Mit Blick auf den Vorschlag von Pfetsch, Löblich und Eilders könnte in diesem Zusammenhang etwa versucht werden, journalistische Praktiken der Produktion von bzw. des Umgangs mit Dissonanzen zu identifizieren. Schließlich hat der Kultursoziologe Andreas Reckwitz (2017) mit seinem jüngsten Entwurf einer ,Gesellschaft der Singularitäten“ ein Theorieangebot gemacht, in das sich eine praxistheoretische Journalistik gesellschaftstheoretisch einbetten ließe.

Aus eher methodologischer Perspektive befasst sich der Beitrag „,Digitale Perspektiven in der Kommunikations- und Mediengeschichte“ von Erik Koenen mit den Dimensionen, Folgen, Prozessen und Grenzen des digitalen Medienwandels für die Quellenarbeit und -kritik in der historischen Presseforschung. Das Vorhaben, die Erkenntnispotenziale und Forschungsszenarien der historischen Presseforschung zu überblicken, ist aus unserer Sicht gerade im Kontext dieses Themenhefts relevant. Pointiert verweist der Beitrag zunächst auf die vermeintliche Randstellung historischer Forschung unter digitalen Vorzeichen (im Gegensatz zu anscheinend relevanteren und prominenteren Feldern wie der Erforschung computervermittelter Kommunikation), um in Abgrenzung dazu die fundamentalen Veränderungen der Digitalisierung und computerbasierten Analyse von Quellen zu beschreiben. Koenen bleibt jedoch nicht bei konzeptuellen und methodologischen Überlegungen stehen, sondern befasst sich im nächsten Schritt auch mit Möglichkeiten der empirischen Umsetzung. Im Zentrum steht dabei eine experimentelle Fallstudie zur medialen Resonanz der Russischen Revolution 1917 in der zeitgenössischen australischen und US-amerikanischen Presse. Hier geht es zum einen um die Erhebung und Aufbereitung digitalisierter Zeitungen und die Ausnutzung der mit diesen Digitalisaten verbundenen Funktionalitäten (Metadatenabfragen, Volltextsuchen, Annotationssystemen); zum anderen befasst er sich mit adäquaten analytischen Ansätzen, um das dadurch eröffnete Erkenntnispotenzial zu gebrauchen. Methodisch schlägt Koenen das „distant reading" als quantifizierte Auswertung großer Textmengen und Metadaten mithilfe von Text Mining vor. Ebenso finden Formen der sogenannten „Culturomics“ Beachtung, wobei hier der Schwerpunkt auf der Identifikation kultureller Muster und Trends liegt. Auf diese Weise soll es möglich sein, einen textstatistischen Blick auf makrostrukturelle Phänomene mit einer analytischen Nahsicht auf die Mikroebene 
von Ausdrücken und Phrasen zu verknüpfen. So wird der Bogen zu Vorhaben wie der Digital Methods Initiative (vgl. Rogers 2013) und den Cultural Analytics (vgl. Manovich 2018) geschlagen. Diese Ansätze verweisen darauf, dass die Beschäftigung mit journalistischen Erzeugnissen und davon ausgehenden Diskursen nicht an digitalisierten Quellen haltmacht, sondern vielmehr um das kommunikative Ineinander verschiedener Kommunikationsformen und Darstellungsweisen erweitert werden kann. In solchen Prozessen der „remediation“ und ,premediation“ werden historische Quellen aufgegriffen, verarbeitet und gedeutet (vgl. Bolter und Grusin 2001; Erll und Rigney 2009).

Der fünfte Beitrag dieses Heftes beschreibt schließlich die empirische und interdisziplinäre Anwendung einer innovativen digitalen Methode auf Fragen der Medienökonomie unter Einbeziehung kommunikationswissenschaftlicher Konzepte. Unter der Überschrift „Der Wert der Worte - Wie digitale Methoden helfen, Kommunikations- und Wirtschaftswissenschaft zu verknüpfen " kombinieren Henrik Müller, Gerret von Nordheim, Karin Boczek, Lars Koppers und Jörg Rahnenführer die theoretischen Konzepte „Frame“ und „Narrativ“ und zeigen am Beispiel des Topic-Modelling-Verfahrens Tendenzen und Trends medialer Berichterstattung auf. Ihre Fallstudie untersucht exemplarisch die Frequenz der Darstellung von wirtschaftlicher Unsicherheit in der Presseberichterstattung im Zeitverlauf und bestimmt Frequenzveränderungen mit dem Rückgriff auf zeithistorische Frames bzw. Narrative. Mit ihrem Vorgehen greifen sie den im Call for Papers formulierten Wunsch nach Beiträgen auf, die zeigen können, wie Kommunikationswissenschaft im interdisziplinären Dialog mit anderen Disziplinen zusammenzuarbeiten vermag. Dazu führt der Beitrag zwei theoretische Konzepte aus der Kommunikations- und der Wirtschaftswissenschaft zusammen, um diese anhand einer empirischen Studie mit interdisziplinärer Anwendung digitaler Methoden konkret zu veranschaulichen. Wir verstehen dies deshalb als sinnvolle Ergänzung zu den übrigen Artikeln des Themenheftes, da hier nicht nur Ergebnisse präsentiert werden, sondern zugleich auch zum Weiterdenken des Vorgehens eingeladen wird. Die Studie adressiert zentrale an innovative Methoden herangetragene Anforderungen wie Interdisziplinarität, theoretische Weiterentwicklung und praktische Anwendbarkeit. Gleichzeitig macht der Beitrag aber auch deutlich, dass der begrenzte Rahmen eines Zeitschriftenartikels selten ausreicht, um einen methodischen Ansatz in der nötigen Tiefe darzustellen. Wir verstehen diesen Beitrag daher auch als Einladung an das Fachkollegium, sich auch über die Herausforderung der angemessenen Darstellung solcher Forschung verstärkt auszutauschen.

Die Zusammenstellung dieser fünf Beiträge kann nur ein vergleichsweise kleiner Ausschnitt aus der Gegenwart zukünftiger Kommunikationswissenschaft sein, sie veranschaulicht aus unserer Sicht aber dennoch sehr gut, wie heterogen die Bereiche sind, die von der geführten Diskussion berührt werden - und welche speziellen Fragen damit jeweils verbunden sind. So meinen wir, dass dieses Themenheft die Vielfalt an theoretischen, methodischen und inhaltlichen Zukünften der Kommunikations- und Medienwissenschaft sehr gut widerspiegelt, und hoffen, dass es auf dieser konkreten Ebene zur weiteren Diskussion anregt. Bedanken möchten wir uns an dieser Stelle bei den Herausgeberinnen und Herausgebern der Publizistik für ihre Einladung und die Möglichkeit zu dieser Co-Herausgeberschaft; bei allen 
Kolleginnen und Kollegen, die auf unseren Call for Papers mit einer Einreichung reagiert haben; bei den Gutachterinnen und Gutachtern, die zu diesem Themenheft wesentlich beigetragen haben; und schließlich bei Gunter Reus für die jederzeit responsive und konstruktive Zusammenarbeit. Wir hoffen, dem Themenheft ist die damit verbundene Arbeit anzumerken, und wünschen allen Leserinnen und Lesern eine gewinnbringende Lektüre.

\section{Literatur}

Bolter, J. D., \& Grusin, R. (2001). Remediation. Cambridge: MIT Press.

Bourdieu, P. (1998). Praktische Vernunft. Frankfurt am Main: Suhrkamp.

Brosius, H.-B. (2016). Warum Kommunikation im Internet öffentlich ist. Zu Andreas Hepps Beitrag „Kommunikations- und Medienwissenschaft in datengetriebenen Zeiten“. Publizistik, 61, 363-372.

Erll, A., \& Rigney, A. (Hrsg.). (2009). Mediation, remediation and the cultural dynamics of memory. Berlin: de Gruyter.

Gherardi, S. (2012). How to conduct a practice-based study. Cheltenham: Edward Elgar.

Hagen, S., Frey, F., \& Koch, S. (2015). Theoriebildung in der Kommunikationswissenschaft. Eine Bestandsaufnahme zu Bedeutung, Arten und Verfahren der Theorieentwicklung. Publizistik, 60, $123-146$.

Hepp, A. (2016). Kommunikations- und Medienwissenschaft in datengetriebenen Zeiten. Publizistik, 61, $225-246$.

Jarren, O. (2016). Nicht Daten, sondern Institutionen fordern die Publizistik- und Kommunikationswissenschaft heraus. Zu Andreas Hepps Beitrag „Kommunikations- und Medienwissenschaft in datengetriebenen Zeiten“. Publizistik, 61, 373-383.

Jünger, J. (2018). Unklare Öffentlichkeit. Individuen in Situationen zwischen öffentlicher und nichtöffentlicher Kommunikation. Wiesbaden: Springer VS.

Klinger, U. (2018). Aufstieg der Semiöffentlichkeit: Eine relationale Perspektive. Publizistik, 63, $245-267$.

Krüger, U., \& Meyen, M. (2018). Auf dem Weg in die Postwachstumsgesellschaft. Plädoyer für eine transformative Kommunikationswissenschaft. Ein Beitrag zur Selbstverständnisdebatte im „Forum“ (Publizistik, Heft 3, 2015; Heft 3 und 4, 2016; Heft 3 und 4, 2017; Heft 1, 2018). Publizistik, 63, 341-357.

Manovich, L. (2018). 100 billion data rows per second: media analytics in the early 21 st century. International Journal of Communication, 12, 473-488.

Meyer, J. W., \& Rowan, B. (1977). Institutionalized organizations. Formal structure as myth and ceremony. American Journal of Sociology, 83, 340-363.

Neuberger, C. (2014). Konflikt, Konkurrenz und Kooperation. Interaktionsmodi in einer Theorie der dynamischen Netzwerköffentlichkeit. Medien \& Kommunikationswissenschaft, 62, 567-587.

Neuberger, C. (2017). Die Rückkehr der Masse. Kollektivphänomene im Internet aus Sicht der Massenund Komplexitätstheorie. Medien \& Kommunikationswissenschaft, 65, 550-572.

Papacharissi, Z. (2015). Affective publics. Sentiment, technology, and politics. New York: Oxford University Press.

Reckwitz, A. (2017). Die Gesellschaft der Singularitäten. Zum Strukturwandel der Moderne. Berlin: Suhrkamp.

Rogers, R. (2013). Digital methods. Cambridge: MIT Press.

Schäfer, F., Daniel, A., \& Hillebrandt, F. (Hrsg.). (2015). Methoden einer Soziologie der Praxis. Bielefeld: transcript.

Schweiger, W. (2004). Das sind doch die Computer-Fuzzis... Aviso, o.J.(37), 14.

Strippel, C., Bock, A., Katzenbach, C., Mahrt, M., Merten, L., Nuernbergk, C., Pentzold, C., \& Waldherr, A. (2018a). Call for Papers für ein Themenheft „Herausforderungen der Digitalisierung - Theoretische und methodische Antworten der Kommunikationswissenschaft“". Publizistik, 63, 7-9.

Strippel, C., Bock, A., Katzenbach, C., Mahrt, M., Merten, L., Nuernbergk, C., Puschmann, C., Pentzold, C., \& Waldherr, A. (2018b). Die Zukunft der Kommunikationswissenschaft ist schon da, sie ist nur ungleich verteilt. Eine Kollektivreplik auf Beiträge im „Forum“ (Publizistik, Heft 3 und 4, 2016). Publizistik, 63, 11-27.

Theis-Berglmair, A. M. (2016). Auf dem Weg zu einer Kommunikationswissenschaft. Zu Andreas Hepps Beitrag „Kommunikations- und Medienwissenschaft in datengetriebenen Zeiten“. Publizistik, 61, 385-391. 
Waldherr, A. (2017). Öffentlichkeit als komplexes System. Theoretischer Entwurf und methodische Konsequenzen. Medien \& Kommunikationswissenschaft, 65, 534-549.

Christian Strippel M.A. ist wissenschaftlicher Mitarbeiter am Institut für Publizistik- und Kommunikationswissenschaft der Freien Universität Berlin.

Dr. Annekatrin Bock ist stellvertretende Abteilungsleiterin der Abteilung „Schulbuch als Medium“ am Georg-Eckert-Institut in Braunschweig.

Dr. Christian Katzenbach ist Senior Researcher am Alexander von Humboldt Institut für Internet und Gesellschaft.

PD Dr. Merja Mahrt ist wissenschaftliche Mitarbeiterin am Institut für Sozialwissenschaften der Heinrich-Heine-Universität Düsseldorf.

Lisa Merten M.A. ist wissenschaftliche Mitarbeiterin am Hans-Bredow-Institut für Medienforschung an der Universität Hamburg.

Dr. Christian Nuernbergk ist Akademischer Rat auf Zeit am Institut für Kommunikationswissenschaft und Medienforschung der Ludwig-Maximilians-Universität München.

Dr. Christian Pentzold ist Juniorprofessor am Zentrum für Medien-, Kommunikations- und Informationsforschung ZeMKI der Universität Bremen.

Dr. Annie Waldherr ist Juniorprofessorin am Institut für Kommunikationswissenschaft der Westfälischen Wilhelms-Universität Münster. 